\title{
Whole-Tumor Histogram and Texture Analyses of DTI for Evaluation of IDH1-Mutation and 1p/19q-Codeletion Status in World Health Organization Grade II Gliomas
}

\author{
(D)Y.W. Park, (D). Han, (DS.S. Ahn, (D)Y.S. Choi, (D).H. Chang, (D) S.H. Kim, DS.-G. Kang, (D)E.H. Kim, and (D).-K. Lee \\ \& $\mathrm{O} \equiv \mathrm{E}$
}

\begin{abstract}
BACKGROUND AND PURPOSE: Prediction of the isocitrate dehydrogenase 1 (IDH1)-mutation and 1p/19q-codeletion status of World Health Organization grade Il gliomas preoperatively may assist in predicting prognosis and planning treatment strategies. Our aim was to characterize the histogram and texture analyses of apparent diffusion coefficient and fractional anisotropy maps to determine IDHImutation and $1 \mathrm{p} / 19 \mathrm{q}$-codeletion status in World Health Organization grade II gliomas.
\end{abstract}

MATERIALS AND METHODS: Ninety-three patients with World Health Organization grade II gliomas with known IDHI-mutation and 1p/19q-codeletion status (18 IDH 1 wild-type, 45 IDH 1 mutant and no 1p/19q codeletion, 30 IDH7-mutant and 1p/19q codeleted tumors) underwent DTI. ROIs were drawn on every section of the T2-weighted images and transferred to the ADC and the fractional anisotropy maps to derive volume-based data of the entire tumor. Histogram and texture analyses were correlated with the IDHI-mutation and $1 \mathrm{p} / 19 \mathrm{q}$-codeletion status. The predictive powers of imaging features for IDHI wild-type tumors and 1p/19q-codeletion status in IDHImutant subgroups were evaluated using the least absolute shrinkage and selection operator.

RESULTS: Various histogram and texture parameters differed significantly according to IDH7-mutation and lp/19q-codeletion status. The skewness and energy of ADC, 10th and 25th percentiles, and correlation of fractional anisotropy were independent predictors of an IDHI wild-type in the least absolute shrinkage and selection operator. The area under the receiver operating curve for the prediction model was 0.853. The skewness and cluster shade of ADC, energy, and correlation of fractional anisotropy were independent predictors of a 1p/19q codeletion in IDHI-mutant tumors in the least absolute shrinkage and selection operator. The area under the receiver operating curve was 0.807 .

CONCLUSIONS: Whole-tumor histogram and texture features of the ADC and fractional anisotropy maps are useful for predicting the IDHI-mutation and $1 \mathrm{p} / 19 \mathrm{q}$-codeletion status in World Health Organization grade II gliomas.

ABBREVIATIONS: FA = fractional anisotropy; GLCM = gray level co-occurrence matrices; IDH = isocitrate dehydrogenase; IDM = inverse different moment; LASSO $=$ least absolute shrinkage and selection operator; $\mathrm{WHO}=$ World Health Organization

$T^{\prime}$ he World Health Organization (WHO) classification of gliomas was updated in 2016. ${ }^{1}$ For the diagnosis of WHO grade II gliomas, the isocitrate dehydrogenase (IDH)-mutation and $1 \mathrm{p} /$ $19 \mathrm{q}$-codeletion status are used in combination with the histologic phenotype; the genotype takes precedence over the histologic

Received October 22, 2017; accepted after revision December 29.

From the Department of Radiology (Y.W.P.), Ewha Womans University College of Medicine, Seoul, Korea; and Departments of Radiology and Research Institute of Radiological Science (Y.W.P., K.H., S.S.A., Y.S.C., S.-K.L.), Neurosurgery (J.H.C., S.-G.K., E.H.K.), and Pathology (S.H.K.), Yonsei University College of Medicine, Seoul, Korea.

This work was supported by the Basic Science Research Program through the National Research Foundation of Korea funded by the Ministry of Science, Information and Communication Technologies, and Future Planning (2017RIDIA1B03030440)

Please address correspondence to Sung Soo Ahn, MD, PhD, Department of Radiology and Research Institute of Radiological Science, Yonsei University College of Medicine, 50-one Yonsei-ro, Seodaemun-gu, Seoul 120-752, Korea; e-mail: sungsoo@yuhs.ac. phenotype in discordant cases. The molecular subtypes of WHO grade II gliomas are divided into 3 classes: $I D H$ wild-type, $I D H$ mutant without $1 \mathrm{p} / 19 \mathrm{q}$ codeletion, and IDH mutant with $1 \mathrm{p} / 19 \mathrm{q}-$ codeletion. ${ }^{1}$ These molecular subtypes reportedly vary in prognosis and chemosensitivity. ${ }^{2}$ Patients with grade II astrocytomas and $I D H$ wild-type have significantly poorer prognoses than those with $I D H$-mutant tumors; the 5-year progression-free survival and overall survival rates are $14 \%$ and $51 \%$ versus $42 \%$ and $93 \%$, respectively. ${ }^{3}$ Moreover, patients with gliomas with $I D H$ mutations were more sensitive to chemoradiation therapy and had longer survival than those with the $I D H$ wild-type. ${ }^{4}$ Additionally,

\footnotetext{
-- Indicates open access to non-subscribers at www.ajnr.org.

$\equiv$ Indicates article with supplemental on-line tables.

Indicates article with supplemental on-line photos.

http://dx.doi.org/10.3174/ajnr.A5569
}

AJNR Am J Neuroradiol 39:693-98 Apr 2018 www.ajnr.org 


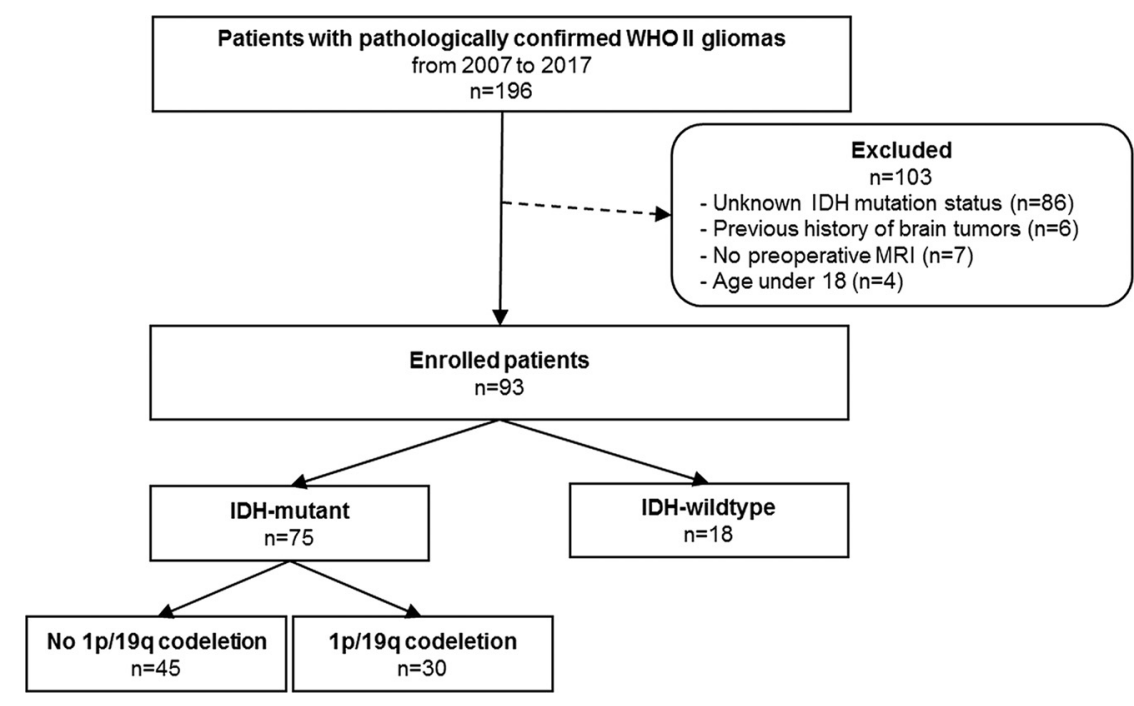

FIGURE. Flowchart of the study population.

$1 \mathrm{p} / 19 \mathrm{q}$ codeletion predicts therapeutic response and survival. ${ }^{5}$ Thus, predicting the IDH-mutation and $1 \mathrm{p} / 19 \mathrm{q}$-codeletion status of WHO grade II gliomas preoperatively may aid in predicting prognosis and planning treatment strategies.

IDH mutation and $1 \mathrm{p} / 19$ codeletion may reflect alterations in tumor cell proliferation and microvessel density that exhibit characteristic features on ADC and fractional anisotropy (FA) parameters. The ADC is an index of tumor cellularity, reflecting tumor burden and correlating negatively with glioma grade. ${ }^{1,6}$ DTI provides information about the motion of water protons at the cellular level ${ }^{7}$; the directional restriction of water diffusivity can be measured as the FA, which correlates with myelinated fiber tract integrity. ${ }^{8,9}$ ADC and FA histograms may predict histologic subtypes, such as oligodendroglioma, oligoastrocytoma, and astrocytoma in WHO grade II gliomas. ${ }^{10,11}$ However, these studies did not obtain molecular markers, and mean or histogram percentile values of ADC and FA have limitations in terms of evaluation of gliomas, due to the heterogeneity. ${ }^{12}$ Histogram-based measures can reflect the intensity distribution of a volume of interest, but not the spatial distribution of these intensities. ${ }^{13}$ On the other hand, texture analysis features show the characteristics of the entire tumor and allow noninvasive quantification of tumor features, such as tumor uniformity, heterogeneity, smoothness, randomness, and symmetry. ${ }^{14}$

To the best of our knowledge, there have been no previous reports that have comprehensively examined the whole-tumor histogram and texture features (ie, ADC and FA parameters), according to the $I D H$-mutation and $1 \mathrm{p} / 19 \mathrm{q}$-codeletion status of grade II gliomas. This study evaluated the role of histogram and texture analyses of ADC and FA maps based on the entire tumor volume in determining the $I D H$-mutation and $1 \mathrm{p} / 19 \mathrm{q}$-codeletion status of grade II gliomas, preoperatively.

\section{MATERIALS AND METHODS Patient Population}

The institutional review board waived the need for obtaining informed patient consent for this retrospective study. Between January 2007 and February 2017, one hundred ninety-six patients with pathologically diagnosed WHO grade II gliomas were included in this study. Inclusion criteria were as follows: 1) WHO grade II gliomas confirmed by histopathology; and 2) patients who underwent preoperative MR imaging, including DTI, with a b-value of 600 $\mathrm{s} / \mathrm{mm}^{2}$. Exclusion criteria were the following: 1) an unknown IDH1-mutation status $(n=86), 2)$ patients with a previous history of brain tumor $(n=6), 3)$ patients with incomplete MR imaging sequences or suboptimal images $(n=7)$, and 4) patients younger than 18 years of age $(n=4)$. The flow chart of the study population is shown in the Figure. The mean interval between the MR imaging examination and the operation was $10.73 \pm 12.49$ days.

Immunohistochemical analysis and peptide nucleic acid-mediated clamping polymerase chain reaction were performed to detect the IDH1 R132H mutation. ${ }^{15}$ For immunohistochemistry, monoclonal antibody $\mathrm{H} 09$ was used, and the degree of IDH1-R132H staining was determined as positive in patients with any stained cells or as negative in those without any stained cells. ${ }^{16}$ If immunohistochemistry results were negative for IDH1-R132H, we confirmed the IDH1 status by peptide nucleic acid-mediated clamping polymerase chain reaction. Fluorescence in situ hybridization analysis was used to investigate $1 \mathrm{p} / 19 \mathrm{q}$ codeletion. ${ }^{17}$

\section{MR Imaging Protocol}

Preoperative MR imaging was performed with a 3T MR imaging scanner (Achieva; Philips Healthcare, Best, the Netherlands) with an 8-channel sensitivity encoding head coil. The preoperative MR imaging protocol included T1-weighted (TR/TE, 1800-2000/ 10-15 ms; FOV, $240 \mathrm{~mm}$; section thickness, $5 \mathrm{~mm}$; matrix, $256 \times$ 256), T2-weighted (TR/TE, 2800-3000/80-100 ms; FOV, 240 $\mathrm{mm}$; section thickness, $5 \mathrm{~mm}$; matrix, $256 \times 256$ ), and FLAIR (TR/TE, 9000-10,000/110-125 ms; FOV, 240 mm; section thickness, $5 \mathrm{~mm}$; matrix, $256 \times 256)$ sequences. 3D contrast-enhanced T1-weighted images (TR/TE, 6.3-8.3/3.1-4 ms; FOV, 240 mm; section thickness, $1 \mathrm{~mm}$; matrix, $192 \times 192$ ) were acquired after administering $0.1 \mathrm{~mL} / \mathrm{kg}$ of gadolinium-based contrast material (gadobutrol, Gadovist; Bayer Schering Pharma, Berlin, Germany). Whole-brain DTI was performed with b-values of 600 and $0 \mathrm{~s} / \mathrm{mm}^{2}, 32$ directions, and the following parameters: TR/TE, 8413.4/77 ms; FOV, 220 mm; section thickness, 2 mm; matrix, $112 \times 112$; acceleration factor, 2.5 ; and with an acquisition time of 5 minutes 20 seconds.

\section{Image Postprocessing: Volume Acquisition}

DTI data were processed off-line with the Medical Image Processing, Analysis, and Visualization software package, Version 7.0 (National Institutes of Health; https://mipav.cit.nih.gov/). T2weighted images were coregistered to ADC and FA maps using affine transformation with normalized mutual information as a cost function, ${ }^{18,19}$ and the ROIs were drawn on every tumor 
Table 1: Patient characteristics according to the IDH1-mutation status and 1p/19q-codeletion status ${ }^{\mathrm{a}}$

\begin{tabular}{|c|c|c|c|}
\hline & $\begin{array}{l}\text { IDHI Wild-Type } \\
\quad(n=18)\end{array}$ & $\begin{array}{l}\text { IDH1 Mutant without 1p/19q } \\
\text { Codeletion }(n=45)\end{array}$ & $\begin{array}{l}\text { IDHI Mutant with } 1 p / 19 q \\
\text { Codeletion }(n=30)\end{array}$ \\
\hline Age (yr) (mean) & $49.57 \pm 3.45$ & $40.80 \pm 1.25$ & $45.10 \pm 1.91$ \\
\hline \multicolumn{4}{|l|}{ Sex } \\
\hline Male & $8(44.4)$ & $27(60)$ & $13(43.3)$ \\
\hline Female & $10(55.6)$ & $18(40)$ & $17(56.7)$ \\
\hline Karnofsky performance status (mean) & $88.89 \pm 1.37$ & $90.67 \pm 1.12$ & $91.00 \pm 1.39$ \\
\hline \multicolumn{4}{|l|}{ Extent of resection } \\
\hline Gross total resection & $7(38.9)$ & $29(64.5)$ & $15(50)$ \\
\hline Subtotal/partial resection & $7(38.9)$ & $14(31.1)$ & $14(46.7)$ \\
\hline Biopsy & $4(22.2)$ & $2(4.4)$ & $1(3.3)$ \\
\hline
\end{tabular}

a Unless otherwise indicated, data are presented as number of patients (\%).

section on T2-weighted images using a semiautomatic method, with signal intensity thresholds as previously described. ${ }^{20}$ The ROIs, were drawn by a single neuroradiologist (Y.W.P) and confirmed by another neuroradiologist (S.S.A.) and were transferred to ADC and FA maps.

\section{Histogram and Texture Analysis of ADC and FA}

First-Order Statistics Including Histogram Parameters. ADC and FA histogram parameters consisting of the mean value and SD were calculated from ROIs overlaid on ADC and FA maps. For cumulative ADC and FA histograms, the 10th, 25th, 50th, 75th, and 90th percentile ADC and FA values in the histogram were generated. ${ }^{10}$ Skewness and kurtosis were also calculated.

Second-Order Statistics via Gray Level Co-Occurrence Matrices. Texture analysis via gray level co-occurrence matrices (GLCM) allows extraction of second-order statistical texture features from images. ${ }^{21} \mathrm{Co}$-occurrence matrix texture considers the relationship between 2 pixels, the reference and neighboring pixel, reflecting local heterogeneity. We used the following parameters for quantitative analysis of the summation of 2D ROIs: contrast, dissimilarity, inverse different moment 1 (IDM1), homogeneity or IDM2, energy, maximum probability, entropy, mean, variance, $\mathrm{SD}$, correlation, cluster shade, and cluster prominence in ADC and FA maps. Altogether, ADC and FA map features were quantified by various features, including their degrees of uniformity (IDM1, homogeneity [IDM2], angular second moment, and energy), heterogeneity (contrast, dissimilarity, variance, and SD), smoothness (correlation), randomness (entropy), and symmetry (cluster shade and prominence). To obtain rotational invariant features, we computed the co-occurrence matrix by averaging $>4$ uniformly distributed angular directions $\left(0^{\circ}, 45^{\circ}\right.$, $90^{\circ}$, and $135^{\circ}$ ).

\section{Statistical Analysis}

The differences between ADC and FA histogram parameters and IDH1-mutation status were assessed using the Student $t$ test or the Mann-Whitney $U$ test, according to normality test results. Because the number of significant imaging features was relatively large, compared with the number of patients when comparing the IDH1 wild-type and IDH1-mutant groups, we used the regularization method to assess the predictive power of the imaging features based on the least absolute shrinkage and selection operator (LASSO), which reduces the potential risk of overfitting or false discovery. LASSO involves penaliz-
Table 2: Prediction model for an IDH1-mutation status in WHO grade II gliomas using the LASSO procedure

\begin{tabular}{lc} 
Imaging Parameters & $\begin{array}{c}\text { Adjusted OR for IDHI } \\
\text { Wild-Type }\end{array}$ \\
\hline ADC skewness & 1.04 \\
ADC energy & 1.15 \\
FA 10th percentile & 23.19 \\
FA 25th percentile & 998.91 \\
FA correlation & 0.004 \\
\hline
\end{tabular}

ing irrelevant variables to zero and retains only useful features, thereby effectively reducing the number of variables. We used 5 -fold cross-validation to find the optimal regularization parameter for LASSO. We estimated the area under the receiver operating curve to assess the predictive ability of variables by selecting significant variables based on LASSO. The 5-fold cross-validated area under the receiver operating curve is the average of the predictive areas under the receiver operating characteristic curve of 5 validation datasets generated by the cross-validation process.

The identical process was performed to evaluate correlations between $\mathrm{ADC}$ and FA histograms and 1p/19q-codeletion status in the IDH1-mutant subgroup.

Statistical analysis was performed using R statistical and computing software (Version 3.3.1; http://www.r-project.org/). Statistical significance was set at $P<.05$.

\section{RESULTS}

The characteristics of the 93 enrolled patients with WHO grade II gliomas are summarized in Table 1. Eighteen patients had IDH1 wild-type tumors, 45 had IDH1-mutant without 1p/19qcodeletion tumors, and 30 had IDH1-mutant with $1 \mathrm{p} / 19 \mathrm{q}-$ codeletion tumors.

\section{Histogram and Texture Analyses of ADC and FA Values for Determining IDH1-Mutation Status}

Various histogram and texture analyses features differed significantly between the IDH1 wild-type and IDH1-mutant groups (On-line Table 1). Among these factors, 5 were independently associated with predicting the IDH1 mutation based on the LASSO procedure (Table 2). The IDH1 wild-type group had higher skewness and energy of ADC and FA 10th and 25th percentiles, whereas the IDH1-mutant group had a higher FA correlation. The area under the receiver operating curve for the optimal model was 0.853 (95\% confidence interval, 0.761-0.945). 
Table 3: Prediction model for $1 \mathrm{p} / 19 \mathrm{q}$-codeletion status in IDHImutant WHO grade II gliomas using the LASSO procedure

Adjusted OR for

Imaging Parameters

$1 \mathrm{p} / 19 \mathrm{q}$ Codeletion

ADC skewness

1.71

ADC cluster shade

1.00

FA energy

0.005

FA correlation

0.12

\section{Histogram and Texture Analyses of ADC and FA Values for Determining $1 p / 19 q$-Codeletion Status in the IDH1-Mutant Group}

Various histogram and texture analysis features were significantly different according to the $1 \mathrm{p} / 19 \mathrm{q}$-codeletion status in the $I D H 1$ mutant subgroup (On-line Table 2). Among these factors, 4 were independently associated with predicting the $1 \mathrm{p} / 19 \mathrm{q}$-codeletion status on the basis of the LASSO procedure (Table 3). The 1p/19qcodeletion group had higher ADC skewness and cluster shade, whereas the group without $1 \mathrm{p} / 19 \mathrm{q}$ codeletion had higher FA energy and FA correlation. The area under the receiver operating curve for the optimal model was 0.807 ( $95 \%$ confidence interval, 0.649-0.965). Representative cases according to IDH1-mutation and $1 \mathrm{p} / 19 \mathrm{q}$-codeletion status are demonstrated in On-line Fig 1. On-line Fig 2 shows the heat map according to IDH1-mutation and $1 \mathrm{p} / 19 \mathrm{q}$-codeletion status, which reveals the strong relationship between significant histogram and texture features of ADC and FA maps $(P<.05)$ and molecular subtypes.

\section{DISCUSSION}

WHO grade II gliomas are heterogeneous at both genetic and histopathologic levels, with intratumoral spatial variation ${ }^{22}$; we comprehensively analyzed the histogram and texture features of whole tumors using ADC and FA maps according to the molecular subtypes.

ADC and FA histogram analyses differ significantly according to histopathologic subtypes or molecular markers of WHO grade II/III gliomas. ${ }^{10,23,24}$ However, some study groups followed the 2007 WHO classification, and texture analyses have not been performed in all studies. Spatial textures in the ADC and FA signals arise from destruction of normal anatomy by tumors, vasogenic edema, tumor cellularity, degenerative changes, or the compression of normal structures, including some that are imperceptible to human eyes. The notion that texture analysis can reveal visually imperceptible tumor information extends beyond radiology to histopathology; texture analysis (in conjunction with histopathology) has been reported to be more accurate than histopathology alone in predicting prognosis in malignant gliomas. ${ }^{25}$ In WHO grade II gliomas, $I D H$ wild-type tumors showed lower $\mathrm{ADC}$ and higher FA values than IDH1-mutant tumors, in agreement with our results. ${ }^{11,23,26}$ Generally, increased tumor cell proliferation and angiogenesis increase tumor cellularity, ${ }^{27}$ and the diffusivity of water molecules is restricted in environments with high cellular density, resulting in lower ADCs. ${ }^{28}$ Therefore, this finding suggests that $I D H$ mutation is associated with lower tumor cellularity ${ }^{29}$ and explains why the presence of an IDH mutation is a favorable prognostic marker in patients with glioma. ${ }^{2}$

DTI-based FA values have received attention in the detection of glioma infiltration. ${ }^{30}$ Previous studies have suggested that tu- mors with higher FA values have higher tumor cell density and a relatively high Ki-67 index in malignant brain tumors, which indicate greater malignancy potential. ${ }^{30,31}$ Therefore, the mechanisms underlying lower FAs in WHO grade II gliomas with $I D H$ mutations may involve a lower rate of proliferation and aggressiveness and lower tumor cell densities of these tumors. In our study, various ADC and FA histograms and texture features differed statistically significantly according to IDH1-mutation status; this finding is useful for enhancing the understanding of tumor heterogeneity according to IDH1-mutation status. The IDH1 wild-type group had higher skewness and energy of ADC and 10th and 25th percentiles of FA, whereas the IDH1-mutant group demonstrated higher FA correlation. The energy represents the orderliness of the image; when the image is highly organized, the energy values are high. Correlation is a measure of the linear dependence of gray levels on those of neighboring pixels (ie, local gray-level dependence); higher values can be obtained for similar gray-level regions. Thus, the $I D H 1$ wild-type group demonstrated higher ADC orderliness, whereas the IDH1-mutant group had a higher frequency of similar FA value regions. Few studies have analyzed the texture features of $\mathrm{T} 1$ contrast-enhanced or $\mathrm{T} 2$ FLAIR images according to the IDH1-mutation status of WHO grade II gliomas ${ }^{32,33}$; but none performed DTI analyses, which provide insight into the spatial distribution of the tumor cellularity and myelinated fiber tract integrity.

In a recent study, diffusion characteristics from visual assessment were significantly different according to the $1 \mathrm{p} / 19 \mathrm{q}$-codeletion status in IDH1-mutant grade II gliomas. ${ }^{34}$ For further evaluation of the roles of diffusion characteristics in determining the $1 \mathrm{p} / 19 \mathrm{q}$-codeletion status of WHO grade II gliomas, we evaluated histogram and texture features measured quantitatively, which can be more objective. Our results demonstrate that histogram and texture features can be effective for predicting $1 \mathrm{p} / 19 \mathrm{q}$-codeletion status. The $1 \mathrm{p} / 19 \mathrm{q}$-codeletion group demonstrated higher ADC skewness, whereas the $1 \mathrm{p} / 19 \mathrm{q}$-intact group had higher FA energy and FA correlation. Thus, the $1 \mathrm{p} / 19 \mathrm{q}$-codeletion group showed more ADC asymmetry, whereas the 1p/19q-intact group had higher FA organization and more regions with similar FA values. Previous studies showed discrepant results of association between $1 \mathrm{p} / 19 \mathrm{q}$ codeletion and ADC and/or FA features in WHO grade II gliomas, ${ }^{24,26,35,36}$ which may be due to different study groups, smaller sample sizes, and different imaging analyses. Several studies included oligoastrocytic or oligodendroglial tumors based on histopathology, according to the 2007 WHO classification, regardless of $I D H$-mutation status; then the authors classified them according to $1 \mathrm{p} / 19 \mathrm{q}$ codeletion status. Therefore, a small number of patients with $I D H$ wild-type gliomas might have been included. Additionally, previous studies included fewer WHO grade II gliomas with evaluation of focal ROIs of the tumor instead of the entire tumor volume.

Because WHO grade II glioma is a heterogeneous tumor with cystic or calcified areas, ADC or FA values calculated in small ROIs cannot represent the cellular density or arrangement of the whole tumor. However, we analyzed tumors according to $1 \mathrm{p} / 19 \mathrm{q}-$ codeletion status in an IDH1-mutant subgroup throughout the whole tumor volume, using comprehensive texture analyses. IDH1-mutant with 1p/19q-codeletion tumors showed lower 
ADC and higher FA values than IDH1-mutant without 1p/19qcodeletion tumors. IDH-mutant with $1 \mathrm{p} / 19 \mathrm{q}$-codeletion tumors are markedly infiltrated by perineuronal satellitosis, with more persistent neurons, as seen on pathology, which may explain their higher FA values. ${ }^{11}$ If the neurons are spared and the volume of invasion is less, edema should be less, which may explain the lower ADC values in such tumors. ${ }^{11}$ Calcification is another pathologic finding related to differences in ADC and FA values according to the 1p/19q-codeletion status in IDH1-mutant tumors; microcalcifications are seen in up to $90 \%$ of $I D H$-mutant with $1 \mathrm{p} / 19 \mathrm{q}$ codeletion tumors. ${ }^{11}$ Tumors with calcifications are expected to have lower ADC values, due to lack of water movement in the calcified region.

Our study had several limitations. First, it was based on a single-center, retrospectively collected dataset. Second, prognostic markers were not analyzed because patients with WHO II gliomas have a relatively long overall survival. Further studies are needed to correlate prognostic markers, such as overall survival and progression-free survival with genotypic and imaging features. Third, $b=600 \mathrm{~s} / \mathrm{mm}^{2}$ was used instead of $b=1000 \mathrm{~s} / \mathrm{mm}^{2}$ or higher in DTI. This low b-value may have resulted in overestimation of ADC values due to perfusion effects. However, this would have little effect on analyzing the difference between molecular groups because the pixels as a whole are affected. Fourth, only IDH1 $\mathrm{R} 132 \mathrm{H}$ mutation was detected in our hospital, and a small proportion of IDH2-mutation or non-IDH1 R132H mutation tumors was not detected. However, it has been previously reported that the predominant amino acid sequence alteration in IDH1 mutation is $\mathrm{R} 132 \mathrm{H}$, accounting for $92.7 \%$ of the detected mutations in WHO grade II and III gliomas. ${ }^{37}$ Fifth, this retrospective study did not investigate the direct relationship between ADC and tumor cellularity from histologic specimens. However, previous studies have proved the negative correlation between ADC and tumor cellularity. ${ }^{38,39}$

\section{CONCLUSIONS}

Histogram and texture features of ADC and FA maps of the entire tumor volume differ according to the IDH1-mutation and 1p/ $19 \mathrm{q}$-codeletion status in WHO grade II gliomas and may be helpful for predicting molecular status.

Disclosures: Sung Soo Ahn—RELATED: Grant: This research received funding from the Basic Science Research Program through the National Research Foundation of Korea funded by the Ministry of Science, Information and Communication Technologies, and Future Planning (2017RID1A1B03030440). * * Money paid to the institution.

\section{REFERENCES}

1. Louis DN, Perry A, Reifenberger G, et al. The 2016 World Health Organization classification of tumors of the central nervous system: a summary. Acta Neuropathol 2016;131:803-20 CrossRef Medline

2. Brat DJ, Verhaak RG, Aldape KD, et al; Genome Atlas Research Network .Comprehensive, integrative genomic analysis of diffuse lower-grade gliomas. $N$ Engl J Med 2015;372:2481-98 CrossRef Medline

3. Metellus P, Coulibaly B, Colin C, et al. Absence of IDH mutation identifies a novel radiologic and molecular subtype of WHO grade II gliomas with dismal prognosis. Acta Neuropathol 2010;120: 719-29 CrossRef Medline

4. Olar A, Wani KM, Alfaro-Munoz KD, et al. IDH mutation status and role of WHO grade and mitotic index in overall survival in grade
II-III diffuse gliomas. Acta Neuropathol 2015;129:585-96 CrossRef Medline

5. Jenkins RB, Blair H, Ballman KV, et al. A t $(\mathbf{1} ; \mathbf{1 9})(\mathbf{q} \mathbf{1 0}$; p10) mediates the combined deletions of $1 p$ and $19 q$ and predicts a better prognosis of patients with oligodendroglioma. Cancer Res 2006;66:9852-61 CrossRef Medline

6. Omuro A, DeAngelis LM. Glioblastoma and other malignant gliomas: a clinical review. JAMA 2013;310:1842-50 CrossRef Medline

7. Balss J, Meyer J, Mueller W, et al. Analysis of the IDH1 codon 132 mutation in brain tumors. Acta Neuropathol 2008;116:597-602 CrossRef Medline

8. Chenevert TL, Brunberg JA, Pipe JG. Anisotropic diffusion in human white matter: demonstration with MR techniques in vivo. $R a$ diology 1990;177:401-05 CrossRef Medline

9. Hansen JR. Pulsed NMR study of water mobility in muscle and brain tissue. Biochim Biophys Acta 1971;230:482-86 CrossRef Medline

10. Tozer DJ, Jäger HR, Danchaivijitr N, et al. Apparent diffusion coefficient histograms may predict low-grade glioma subtype. NMR Biomed 2007;20:49-57 CrossRef Medline

11. Khayal IS, McKnight TR, McGue C, et al. Apparent diffusion coefficient and fractional anisotropy of newly diagnosed grade II gliomas. NMR Biomed 2009;22:449-55 CrossRef Medline

12. Ryu YJ, Choi SH, Park SJ, et al. Glioma: application of whole-tumor texture analysis of diffusion-weighted imaging for the evaluation of tumor heterogeneity. PLoS One 2014;9:e108335 CrossRef Medline

13. Brynolfsson P, Nilsson D, Henriksson R, et al. ADC texture: an imaging biomarker for high-grade glioma? Medical Phys 2014;41: 101903 CrossRef Medline

14. Davnall F, Yip CS, Ljungqvist G, et al. Assessment of tumor heterogeneity: an emerging imaging tool for clinical practice? Insights Imaging 2012;3:573-89 CrossRef Medline

15. Yan H, Parsons DW, Jin G, et al. IDH1 and IDH2 mutations in gliomas. N Engl J Med 2009;360:765-73 CrossRef Medline

16. Takano S, Tian W, Matsuda M, et al. Detection of IDH1 mutation in human gliomas: comparison of immunohistochemistry and sequencing. Brain Tumor Pathol 2011;28:115-23 CrossRef Medline

17. Riemenschneider MJ, Jeuken JW, Wesseling P, et al. Molecular diagnostics of gliomas: state of the art. Acta Neuropathol 2010;120: 567-84 CrossRef Medline

18. Cha J, Kim S, Kim HJ, et al. Differentiation of tumor progression from pseudoprogression in patients with posttreatment glioblastoma using multiparametric histogram analysis. AJNR Am J Neuroradiol 2014;35:1309-17 CrossRef Medline

19. Maes F, Collignon A, Vandermeulen D, et al. Multimodality image registration by maximization of mutual information. IEEE Trans Med Imaging 1997;16:187-98 CrossRef Medline

20. Ko SB, Choi HA, Carpenter AM, et al. Quantitative analysis of hemorrhage volume for predicting delayed cerebral ischemia after subarachnoid hemorrhage. Stroke 2011;4:669-74 CrossRef Medline

21. Haralick RM, Shanmugam K. Textural features for image classification. IEEE Trans Syst Man Cybern 1973;610-21 CrossRef

22. Paulus W, Peiffer J Intratumoral histologic heterogeneity of gliomas: a quantitative study. Cancer 1989;64:442-47 Medline

23. Xiong J, Tan WL, Pan JW, et al. Detecting isocitrate dehydrogenase gene mutations in oligodendroglial tumors using diffusion tensor imaging metrics and their correlations with proliferation and microvascular density. J Magn Reson Imaging 2016;43:45-54 CrossRef Medline

24. Jenkinson MD, Smith TS, Brodbelt AR, et al. Apparent diffusion coefficients in oligodendroglial tumors characterized by genotype. J Magn Reson Imaging 2007;26:1405-12 CrossRef Medline

25. Zacharaki EI, Morita N, Bhatt P, et al. Survival analysis of patients with high-grade gliomas based on data mining of imaging variables. AJNR Am J Neuroradiol 2012;33:1065-71 CrossRef Medline

26. Xiong J, Tan W, Wen J, et al. Combination of diffusion tensor imaging and conventional MRI correlates with isocitrate dehydrogenase $1 / 2$ mutations but not $1 \mathrm{p} / 19 \mathrm{q}$ genotyping in oligodendroglial tumours. Eur Radiol 2016;26:1705-15 CrossRef Medline 
27. Fischer I, Gagner JP, Law M, et al. Angiogenesis in gliomas: biology and molecular pathophysiology. Brain Pathol 2005;15:297310 Medline

28. Yamasaki F, Kurisu K, Satoh K, et al. Apparent diffusion coefficient of human brain tumors at MR imaging. Radiology 2005;235:985-91 CrossRef Medline

29. Bralten LB, Kloosterhof NK, Balvers R, et al. IDH1 R132H decreases proliferation of glioma cell lines in vitro and in vivo. Ann Neurol 2011;69:455-63 CrossRef Medline

30. Beppu T, Inoue T, Shibata $Y$, et al. Fractional anisotropy value by diffusion tensor magnetic resonance imaging as a predictor of cell density and proliferation activity of glioblastomas. Surg Neurol 2005;63:56-61; discussion 61 CrossRef Medline

31. Kinoshita M, Hashimoto N, Goto T, et al. Fractional anisotropy and tumor cell density of the tumor core show positive correlation in diffusion tensor magnetic resonance imaging of malignant brain tumors. Neuroimage 2008;43:29-35 CrossRef Medline

32. Yu J, Shi Z, Lian Y, et al. Noninvasive IDH1 mutation estimation based on a quantitative radiomics approach for grade II glioma. Eur Radiol 2017;27:3509-22 CrossRef Medline

33. Li Z, Wang Y, Yu J, et al. Deep learning based radiomics (DLR) and its usage in noninvasive IDH1 prediction for low grade glioma. Sci Rep 2017;7:5467 CrossRef Medline
34. Leu K, Ott GA, Lai A, et al. Perfusion and diffusion MRI signatures in histologic and genetic subtypes of WHO grade II-III diffuse gliomas. J Neurooncol 2017;134:177-88 CrossRef Medline

35. Khayal IS, Vandenberg SR, Smith KJ, et al. MRI apparent diffusion coefficient reflects histopathologic subtype, axonal disruption, and tumor fraction in diffuse-type grade II gliomas. Neuro Oncol 2011; 13:1192-201 CrossRef Medline

36. Park Y, Han K, Ahn S, et al. Prediction of IDH1-mutation and 1p/ 19q-codeletion status using preoperative MR imaging phenotypes in lower grade gliomas. AJNR Am J Neuroradiol 2018;39:37-42 CrossRef Medline

37. Hartmann C, Meyer J, Balss J, et al. Type and frequency of IDH1 and IDH2 mutations are related to astrocytic and oligodendroglial differentiation and age: a study of 1,010 diffuse gliomas. Acta Neuropathol 2009;118:469-74 CrossRef Medline

38. Kono K, Inoue $\mathrm{Y}$, Nakayama K, et al. The role of diffusion-weighted imaging in patients with brain tumors. AJNR Am J Neuroradiol 2001;22:1081-88 Medline

39. Sugahara T, Korogi Y, Kochi M, et al. Usefulness of diffusionweighted MRI with echo-planar technique in the evaluation of cellularity in gliomas. J Magn Reson Imaging 1999;9:53-60 Medline 